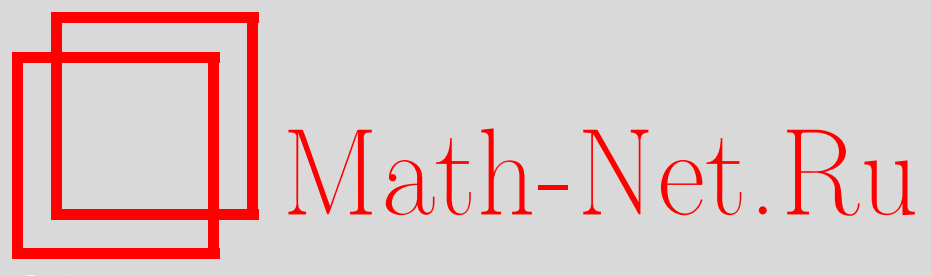

В. Балаж, К. Нагасака, О. Штраух, Закон Бенфорда и функции распределения последовательностей на $(0,1)$, Матем. заметки, 2010, том 88, выпуск 4, 485-501

DOI: https://doi.org/10.4213/mzm8848

Использование Общероссийского математического портала Math-Net.Ru подразумевает, что вы прочитали и согласны с пользовательским соглашением http://www . mathnet.ru/rus/agreement

Параметры загрузки:

IP : 54.210 .77 .194

26 апреля 2023 г., 13:10:25

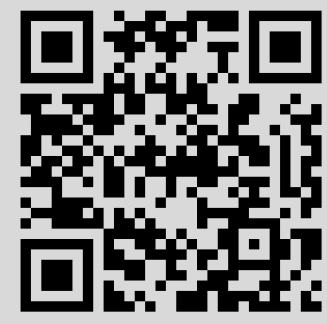


Том 88 выпуск 4 октябрь 2010

\author{
УДК 517
}

\title{
Закон Бенфорда и функции распределения последовательностей на $(0,1)$
}

\section{В. Балаж, К. Нагасака, О. Штраух}

Применяя теорию функций распределения последовательностей $x_{n} \in[0,1]$, $n=1,2, \ldots$, мы находим функциональное уравнение для функции распределения последовательности $x_{n}$, а то, что последовательность $x_{n}$ удовлетворяет функциональному уравнению, эквивалентно тому, что $x_{n}$ удовлетворяет закону Бенфорда в сильной форме. Даны примеры функций распределения последовательностей, удовлетворяющих функциональному уравнению, с применением для сильного закона Бенфорда для разных оснований. Показаны некоторые непосредственные следствия из теории равномерного распределения для сильного закона Бенфорда.

Библиография: 14 названий.

\section{Посвящается памяти Анатолия Алексеевича Карацубъ}

1. Введение. Проблема первой цифры: бесконечная последовательность $x_{n} \geqslant 1$ вещественных чисел удовлетворяет закону Бенфорда, если частота (асимптотическая плотность) появления значащей цифры $a$ (где $x_{n}$ записано в десятичном виде) первой, удовлетворяет формуле $\log _{10}(1+1 / a)$ для каждой $a=1,2, \ldots, 9$ (0 в качестве возможной первой цифры не допускается). Поскольку $x_{n}$ имеет первой цифру $a$ тогда и только тогда, когда

$$
\log _{10} x_{n} \bmod 1 \in\left[\log _{10} a, \log _{10}(a+1)\right),
$$

закон Бенфорда для $x_{n}$ следует из равномерного распределения для $\log _{10} x_{n} \bmod 1$.

Ньюкомб [1] первым заметил, что "то, что десять иифр не случаются с равной частотой долюно быть очевидно каждому, пользующемуся логарифмическими таблииами". Бенфорд [2] сравнивал эмпирическую частоту появления а со значением $\log _{10}(1+1 / a)$ по двадцати различным таблицам, длина которых колебалась в пределах от 91 входных данных (таблица атомных весов) до 5000 входных данных (в математическом справочнике), что привело его к заключению, что "логарифмический закон применим особенно к"внесписочным" числам, которые не связаны какими-либо известными соотношениями ...".

Дж. Циглер предложил (ср. Райми [3]) назвать $x_{n}$ сильной бенфордовой последовательностъю, если $\log _{10} x_{n} \bmod 1$ распределяется равномерно. По Дьяконису [4]

Работа первого автора выполнена при поддержке гранта VEGA No. 1/0374/08; работа третьего автора выполнена при поддержке гранта VEGA No. 2/7138/27.

(C) В. БАЛАЖ, К. НАГасака, О. Штраух, 2010 
последовательность $x_{n}$ определяется как удовлетворяющая сильному закону Бенфорда, если для любого превоначального блока цифр $a_{1} a_{2} \ldots a_{r}$ плотность $a_{1} a_{2} \ldots a_{r}$ равна

$$
\log _{10}\left(1+\frac{1}{a_{1} a_{2} \cdots a_{r}}\right) .
$$

Кунов [5] независимо заметил, что равномерное распределение $\log _{10} n ! \bmod 1$ влечет за собой то, что последовательность $n$ ! удовлетворяет сильному закону Бенфорда, как было сформулировано в [4].

Библиография, приведенная Райми [3], была практически полной до 1976. Другой всесторонний обзор по закону Бенфорда представил Шатте [6], а некоторые результаты на эту тему могут быть найдены в работе Нагасаки, Канемитсу, Шуе [7].

В настоящей статье мы применим к проблеме первой цифры теорию функций распределения последовательностей, основные результаты по которой могут быть найдены в монографиях [8]-[10]. Павлов [11] был первым, кто применил теорию функций распределения, но наша статья предлагает совершенно новые результаты.

В п. 2 мы повторим основные определения и результаты для общего целого основания $b$. В п. 3 мы представим (теорема 9) основное применение теории функций распределения для закона Бенфорда. Мы докажем, что последовательность $x_{n} \in(0,1)$ может удовлетворять "сильному" закону Бенфорда только для конечного числа оснований. В п. 4 мы переформулируем несколько теорем из теории равномерного распределения на языке закона Бенфорда. Отметим, что в конце (пример 10) мы полностью решим проблему асимптотической плотности появления цифр в последовательности всех натуральных чисел $1,2,3, \ldots$, которая не удовлетворяет закону Бенфорда.

2. Основные определения и результаты. Пусть $b \geqslant 2$ есть целое число, которое рассматривается как основание для представления вещественного числа $x>0$, и $M_{b}(x)$ есть мантисса $x, x=M_{b}(x) \times b^{n(x)}$, такая, что $1 \leqslant M_{b}(x)<b$, где $n(x)$ есть однозначно определенное целое число. Пусть $K=k_{1} k_{2} \ldots k_{r}$ есть положительное целое число, представленное по основанию $b$, т.е.

$$
K=k_{1} \times b^{r-1}+k_{2} \times b^{r-2}+\cdots+k_{r-1} \times b+k_{r},
$$

где $k_{1} \neq 0$, и в то же время $K=k_{1} k_{2} \ldots k_{r}$ рассматривается как некоторый блок $r$-последовательных цифр по основанию $b$. Тогда у $x$ первые $r$ цифр равны $k_{1} k_{2} \ldots k_{r}$ только тогда, когда ${ }^{1}$

$$
k_{1} \cdot k_{2} \ldots k_{r} \leqslant M_{b}(x)<k_{1} \cdot k_{2} \ldots\left(k_{r}+1\right) .
$$

Так как $\log _{b} M_{b}(x)=\log _{b} x \bmod 1$, то неравенство (2.1) эквивалентно

$$
\log _{b}\left(\frac{K}{b^{r-1}}\right) \leqslant \log _{b} x \bmod 1<\log _{b}\left(\frac{K+1}{b^{r-1}}\right) .
$$

Заметим, что для $x$ вида $x=0.00 \ldots 0 k_{1} k_{2} \ldots k_{r} \ldots$, мы опустили первые нулевые знаки, и $M_{b}(x)=k_{1} \cdot k_{2} \ldots k_{r} \ldots$.

\footnotetext{
${ }^{1}$ Если $k_{1}=k_{2}=\cdots=k_{r}=b-1$, то $k_{1} \cdot k_{2} \ldots\left(k_{r}+1\right)=b$.
} 
ОПРЕДЕЛЕНИЕ 1 . Последовательность $x_{n}, n=1,2, \ldots$, положительных вещественных чисел удовлетворяет закону Бенфорда (аббревиатура B.L.) порядка $r$, если для любого $r$-значного целого $K=k_{1} k_{2} \ldots k_{r}$ имеем

$$
\lim _{N \rightarrow \infty} \frac{\#\left\{n \leqslant N \text {; первые } r \text { знаков } M_{b}\left(x_{n}\right) \text { равны } K\right\}}{N}=\log _{b}(K+1)-\log _{b} K .
$$

Сошлемся при этом на монографии [8]-[10].

ОПРЕДЕЛЕНИЕ 2. (i) Пусть $x_{n}, n=1,2, \ldots$, есть последовательность вещественных чисел, определим ступенчатую функцию распределения $x_{n} \bmod 1$

$$
F_{N}(x)=\frac{\#\left\{n \leqslant N ; x_{n} \bmod 1 \in[0, x)\right\}}{N}
$$

при $x \in[0,1]$.

(ii) Функция распределения (аббревиатура d.f.) $g(x)$ называется d.f. последовательности $x_{n} \bmod 1, n=1,2, \ldots$, если существует последовательность индексов $N_{1}<N_{2}<\cdots$ такая, что

$$
\lim _{i \rightarrow \infty} F_{N_{i}}(x)=g(x)
$$

при всех $x \in[0,1)$. Здесь используется так называемое сильное определение d.f. (см. [8]), но в [10] предполагается, что предел (2.5) справедлив только для всех точек непрерывности $g(x), x \in[0,1]$.

(iii) Обозначим набор всех функций d.f. последовательности $x_{n} \bmod 1, n=1,2, \ldots$, через $G\left(x_{n} \bmod 1\right)$. Если $G\left(x_{n} \bmod 1\right)$ является синглтоном, т.е. $G\left(x_{n} \bmod 1\right)=\{g(x)\}$, то $g(x)$ называется асимптотической функиией распределения (аббревиатура а.d.f.) $x_{n} \bmod 1$, и если, кроме того, $g(x)=x$, то последовательность $x_{n} \bmod 1$ является равномерно распределенной (аббревиатура u.d.) на $[0,1]$.

(iv) $c_{\alpha}(x)$ есть одноступенчатая d.f., для которой $c_{\alpha}(x)=0$, если $0 \leqslant x<\alpha$ и $c_{\alpha}(x)=1$, если $\alpha \leqslant x \leqslant 1$.

Tеорема 1. Пусть $G\left(\log _{b}\left(x_{n}\right) \bmod 1\right)$ есть набор всех d.f. последовательности $\log _{b}\left(x_{n}\right) \bmod 1, n=1,2, \ldots$. Последовательность $x_{n}$ удовлетворяет B.L. порядка $r$ тогда и только тогда, когда для любого $g(x) \in G\left(\log _{b}\left(x_{n}\right) \bmod 1\right)$ справедливо соотношение

$$
g\left(\log _{b}\left(\frac{K}{b^{r-1}}\right)\right)=\log _{b}\left(\frac{K}{b^{r-1}}\right)
$$

где $K=k_{1} k_{2} \ldots k_{r}$ - произвольное положительное $r$-значное челое число.

ДокаЗАТЕЛЬСтво. Пусть $x_{n}>0, n=1,2, \ldots, g(x) \in G\left(\log _{b}\left(x_{n}\right) \bmod 1\right)$ и

$$
g(x)=\lim _{i \rightarrow \infty} F_{N_{i}}(x), \quad \text { где } \quad F_{N_{i}}(x)=\frac{\#\left\{n \leqslant N_{i} ; \log _{b}\left(x_{n}\right) \bmod 1 \in[0, x)\right\}}{N_{i}} .
$$

Пользуясь эквивалентными неравенствами $(2.2)$ и $(2.1)$, для фиксированного $N_{i}$ имеeм

$$
\begin{aligned}
& \frac{\#\left\{n \leqslant N_{i} ; \text { первые } r \text { знаков } M_{b}\left(x_{n}\right) \text { равны } K\right\}}{N_{i}} \\
& =\frac{\#\left\{n \leqslant N_{i} ; \log _{b}\left(K / b^{r-1}\right) \leqslant \log _{b} x_{n} \bmod 1<\log _{b}\left((K+1) / b^{r-1}\right)\right\}}{N_{i}} \\
& =F_{N_{i}}\left(\log _{b}\left(\frac{K+1}{b^{r-1}}\right)\right)-F_{N_{i}}\left(\log _{b}\left(\frac{K}{b^{r-1}}\right)\right)
\end{aligned}
$$


и (2.3) можно переписать как

$$
\lim _{i \rightarrow \infty}\left(F_{N_{i}}\left(\log \left(\frac{K+1}{b^{r-1}}\right)\right)-F_{N_{i}}\left(\log _{b}\left(\frac{K}{b^{r-1}}\right)\right)\right)=\log _{b}(K+1)-\log _{b} K .
$$

Таким образом,

$$
g\left(\log _{b}\left(\frac{K+1}{b^{r-1}}\right)\right)-g\left(\log _{b}\left(\frac{K}{b^{r-1}}\right)\right)=\log _{b}\left(\frac{K+1}{b^{r-1}}\right)-\log _{b}\left(\frac{K}{b^{r-1}}\right) .
$$

Суммируя (2.8) по всем $r$-значным целым $K^{\prime}, K^{\prime}<K$, находим (2.6).

ЗАмечание 1. Определение 1 и теорема 1 неявно присутствуют в [11].

Теорема 2. Для любого $r, r=1,2, \ldots$, существует последовательность $x_{n}$, $n=1,2, \ldots$, такая, что $x_{n}$ удовлетворяет B.L. порядка $r$, но не удовлетворяет B.L. порядка $r+1$ по основанию $b \geqslant 2$.

ДокАЗАТЕЛЬСтво. Пусть

$$
A_{r}=\left\{\log _{b}\left(k_{1} \cdot k_{2} k_{3} \ldots k_{r}\right) ; k_{i} \in\{0,1, \ldots, b-1\}, i=1,2, \ldots, r, k_{1} \neq 0\right\},
$$

где в этом случае $k_{1} \cdot k_{2} k_{3} \ldots k_{r}=K / b^{r-1}$ и $K$ есть положительное целое, представленное по основанию $b$, т.е.

$$
K=k_{1} \times b^{r-1}+k_{2} \times b^{r-2}+\cdots+k_{r-1} \times b+k_{r} .
$$

Определим d.f. $g(x)$ такую, что $g(x)=x$ для любого $x \in A_{r}$, но $g(x) \neq x$ для некоторого $x \in A_{r+1}$. Согласно известной теореме, для любой d.f. $g(x)$ существует последовательность $y_{n} \in[0,1), n=1,2, \ldots$, такая, что $g(x)$ является a.d.f последовательности $y_{n}$ (см. [8; с. 138]). Поэтому определим последовательность $x_{n}$, как $x_{n}=b^{y_{n}}$, т.е. $\log _{b} x_{n}=y_{n}$, при $n=1,2, \ldots$. Применяя теорему 1 , находим, что $x_{n}$ удовлетворяет B.L. порядка $r$ и не удовлетворяет B.L. порядка $r+1$.

Непосредственно из доказательства теоремы 2 получаем следующее.

СлЕДСтвиЕ 1. Если последовательность $x_{n}, n=1,2, \ldots$, удовлетворяет B.L. порядка $r$, то она одновременно удовлетворяет и B.L. порядка $r^{\prime} \leqslant r$.

ОПРЕДЕЛЕНИЕ 3. Если последовательность $x_{n}, n=1,2, \ldots$, удовлетворяет B.L. порядка $r$ для любого $r=1,2, \ldots$, то $x_{n}$ называется удовлетворяющей сильномy B.L.

Из теоремы 1 непосредственно следует

Теорема 3. Последовательность $x_{n}, x_{n}>0, n=1,2, \ldots$, удовлетворяет сильному B.L. тогда и только тогда, когда последовательность $\log _{b} x_{n} \bmod 1$ является u.d. на $[0,1)$.

ДоказАтельство. Пусть $g(x) \in G\left(\log _{b}\left(x_{n}\right) \bmod 1\right)$. Теорема 1 имеет следствием то, что $g(x)=x$ для всех $x=\log _{b}\left(K / b^{r-1}\right)$. Поскольку набор таких $x$ плотный для $K=k_{1} k_{2} \ldots k_{r}, k_{1} \neq 0,0 \leqslant k_{i}<b, r=1,2, \ldots$, отсюда имеем $g(x)=x$ для всех $x \in[0,1]$.

ЗАмечАние 2. Известная теорема 3 может быть найдена также в [4] и [11]. 


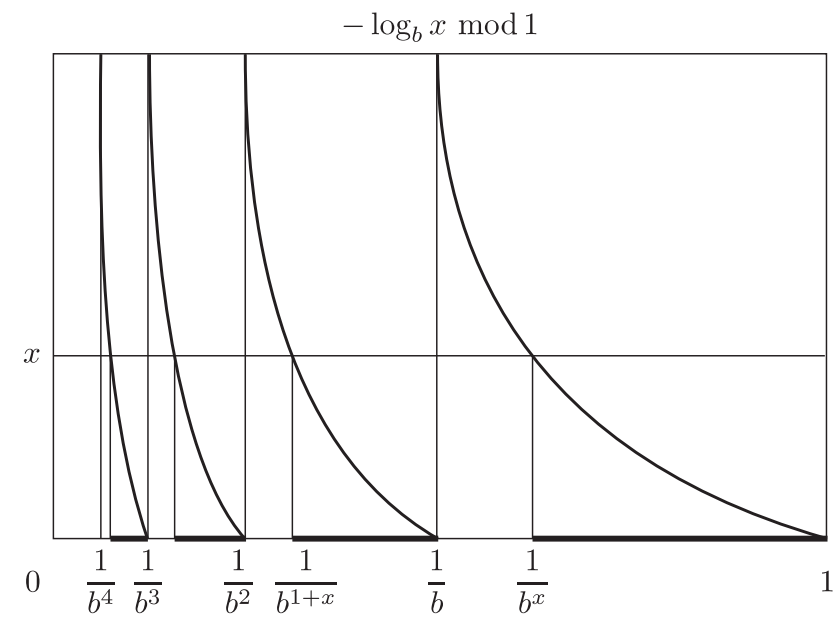

Рис. 1. Интервалы $f_{i}^{-1}([0, x)), i=0,1,2, \ldots$

\section{3. Функции распределения последовательностей из единичного интер-} вала, которые удовлетворяют сильному закону Бенфорда.

Теорема 4. Пусть $x_{n}, n=1,2, \ldots$, - последовательность на $(0,1)$ и $G\left(x_{n}\right)$ набор всех d.f. $x_{n}$. Предположим, что любая d.f. $g(x) \in G\left(x_{n}\right)$ является непрерывной в $x=0$. Тогда последовательность $x_{n}$ удовлетворяет сильному B.L. по основанию $b$ только тогда, когда для любой $g(x) \in G\left(x_{n}\right)$ справедливо соотношение

$$
x=\sum_{i=0}^{\infty}\left(g\left(\frac{1}{b^{i}}\right)-g\left(\frac{1}{b^{i+x}}\right)\right) \quad \text { для } x \in[0,1] .
$$

ДокАЗАтЕЛьство. Прежде всего, заметим, что последовательности $\log _{b} x_{n} \bmod 1$ и $-\log _{b} x_{n} \bmod 1$ являются одновременно u.d., так что далее будем изучать $-\log _{b} x_{n}$ $\bmod 1, n=1,2, \ldots$. Теперь рассмотрим функцию $-\log _{b} x$, определенную в области $(0,1]$. Разделим область $(0,1]$ на бесконечно много частей

$$
\left(\frac{1}{b^{i+1}}, \frac{1}{b^{i}}\right], \quad i=0,1,2, \ldots,
$$

и на каждой части $\left(1 / b^{i+1}, 1 / b^{i}\right]$ введем обозначение

$$
f_{i}(x)=-\log _{b} x \bmod 1=-\log _{b} x-i .
$$

Тогда для любого члена $x_{n} \in(0,1)$ и при $x \in[0,1]$ имеем

$$
0 \leqslant-\log _{b} x_{n} \bmod 1<x \quad \Longleftrightarrow \quad \exists i \in\{0,1,2, \ldots\} \quad\left(x_{n} \in f_{i}^{-1}([0, x))\right),
$$

где

$$
f_{i}^{-1}([0, x))=\left(\frac{1}{b^{i+x}}, \frac{1}{b^{i}}\right] .
$$

Следуя (2.4), обозначим два типа ступенчатых d.f. функций

$$
\begin{aligned}
& F_{N}^{(1)}(x)=\frac{\#\left\{n \leqslant N ;-\log _{b} x_{n} \bmod 1 \in[0, x)\right\}}{N}, \\
& F_{N}^{(2)}(x)=\frac{\#\left\{n \leqslant N ; x_{n} \bmod 1 \in[0, x)\right\}}{N} .
\end{aligned}
$$


Тогда (3.2) имеет вид

$$
F_{N}^{(1)}(x)=\sum_{i=0}^{\infty} F_{N}^{(2)}\left(\frac{1}{b^{i}}\right)-F_{N}^{(2)}\left(\frac{1}{b^{i+x}}\right) .
$$

Кроме того, по определению $F_{N}^{(2)}(x)$

$$
\sum_{i=K}^{\infty} F_{N}^{(2)}\left(\frac{1}{b^{i}}\right)-F_{N}^{(2)}\left(\frac{1}{b^{i+x}}\right) \leqslant F_{N}^{(2)}\left(\frac{1}{b^{K}}\right) .
$$

Теперь предположим, что $g(x) \in G\left(x_{n}\right)$, т.е. существует последовательность индексов $N_{1}<N_{2}<\cdots$ такая, что

$$
\lim _{N_{k} \rightarrow \infty} F_{N_{k}}^{(2)}(x)=g(x) \quad \text { для любого } \quad x \in[0,1] .
$$

Согласно теореме Хелли (см. [10; с. 4-5, теорема 4.1.0.10]) из $N_{k}$ может быть выделена подпоследовательность $N_{k}^{\prime}$ такая, что

$$
\lim _{N_{k}^{\prime} \rightarrow \infty} F_{N_{k}^{\prime}}^{(1)}(x)=\widetilde{g}(x) .
$$

Суммируя (3.3) и (3.4), находим

$$
\left|\widetilde{g}(x)-\sum_{i=0}^{K-1}\left(g\left(\frac{1}{b^{i}}\right)-g\left(\frac{1}{b^{i+x}}\right)\right)\right| \leqslant g\left(\frac{1}{b^{K}}\right)
$$

при $x \in[0,1]$. Непрерывность влечет $g\left(1 / b^{K}\right) \rightarrow 0$ при $K$ стремящемся к бесконечности, и мы имеем

$$
\widetilde{g}(x)=x \quad \Longleftrightarrow \quad x=\sum_{i=0}^{\infty}\left(g\left(\frac{1}{b^{i}}\right)-g\left(\frac{1}{b^{i+x}}\right)\right) .
$$

Завершение доказательства: если $x_{n}$ удовлетворяет сильному закону Бенфорда, т.е. $\widetilde{g}(x)=x$, то (3.1) справедливо для любого $g(x) \in G\left(x_{n}\right)$. И обратно: (3.1) влечет $\widetilde{g}(x)=x$ для любой функции распределения $-\log x_{n} \bmod 1$, поскольку мы можем начать $\mathrm{c}$

$$
\lim _{N_{k} \rightarrow \infty} F_{N_{k}}^{(1)}(x)=\widetilde{g}(x),
$$

а затем выбрать подпоследовательность $N_{k}^{\prime}$ такую, что

$$
\lim _{N_{k}^{\prime} \rightarrow \infty} F_{N_{k}^{\prime}}^{(2)}(x)=g(x) .
$$

ЗАмечаниЕ 3. Непрерывность $g(x)$ в $x=0$ является необходимым условием, так как последовательность $x_{n}=\alpha^{n}, n=1,2, \ldots, 0<\alpha<1$, где $\log _{b} \alpha$ является иррациональным значением, удовлетворяет сильному B.L. по основанию $b$, но $G\left(x_{n}\right)=\left\{c_{0}(x)\right\}$, и одноступенчатая d.f. $c_{0}(x), c_{0}(x)=1$ при $x \in(0,1]$, не удовлетворяет (3.1).

Следующий критерий непрерывности вытекает непосредственно из теоремы 2.1 работы [12]. 
Теорема 5. Произволъная d.f. $g(x) \in G\left(x_{n}\right)$ непрерывна в $x=0$ тогда и только тогда, когда для любой подпоследовательности $x_{n_{k}} \rightarrow 0, k \rightarrow \infty$, асимптотическая плотность $d\left(n_{k}\right)=0$.

В дальнейших примерах 1, 2 и 3 мы найдем некоторые частные решения уравнения (3.1). Это будет основано на следующем построении последовательностей $x_{n}$, удовлетворяющих B.L.: пусть $g(x)$ есть d.f., непрерывная в $x=0$, которая является решением (3.1). Тогда согласно [8; с. 140, теорема 4.4] существует последовательность $x_{n} \in(0,1)$ с a.d.f. $g(x)$, и согласно теореме 4 эта $x_{n}$ удовлетворяет сильномy B.L. Заметим, что если $g_{1}(x), \ldots, g_{k}(x)$ - решения $(3.1)$ и

$$
\alpha_{1}+\cdots+\alpha_{k}=1, \quad \alpha_{i} \geqslant 0, \quad i=1,2, \ldots, k,
$$

Tо

$$
\alpha_{1} g_{1}(x)+\cdots+\alpha_{k} g_{k}(x)
$$

также удовлетворяет (3.1). Наконец, заметим, что из [10; с. 1-9] следует, что если $H$ - непустой, замкнутый и связный набор решений (3.1), непрерывный в $x=0$, то существует последовательность $x_{n} \in(0,1), n=1,2, \ldots$, такая, что $H=G\left(x_{n}\right)$ и эта $x_{n}$ удовлетворяет сильному B.L.

ПримеР 1. Выберем функцию $g(x)$, возрастающую на интервале $[0,1 / b], g(0)=0$; и $g(x)$ на $(1 / b, 1]$ должна удовлетворять

$$
g\left(\frac{1}{b^{x}}\right)=\sum_{i=1}^{\infty}\left(g\left(\frac{1}{b^{i}}\right)-g\left(\frac{1}{b^{i+x}}\right)\right)+1-x .
$$

Определенная таким образом функция $g:[0,1] \rightarrow[0,1]$ будет d.f., если выполняются следующие условия:

(i) $g(x)$ является неубывающей для $x \in(1 / b, 1]$;

(ii) $g(1 / b) \leqslant \lim _{x \rightarrow 1-0} g\left(1 / b^{x}\right)$;

(iii) $\lim _{x \rightarrow 0} g\left(1 / b^{x}\right) \leqslant 1$.

Полагая $g(x)=x$ при $x \in[0,1 / b]$, получим из $(3.7)$

$$
g\left(\frac{1}{b^{x}}\right)=\sum_{i=1}^{\infty}\left(g\left(\frac{1}{b^{i}}\right)-g\left(\frac{1}{b^{i+x}}\right)\right)+1-x=1-x+\left(1-\frac{1}{b^{x}}\right) \frac{1}{b-1} .
$$

Заменяя $1 / b^{x}$ на $x$ в (3.8), находим $g(x)$ при $x \in[1 / b, 1]$, которая удовлетворяет (i)-(iii), и отсюда имеем следующее решение $g(x)$ уравнения $(3.1)$ :

$$
g(x)= \begin{cases}x, & \text { если } x \in\left[0, \frac{1}{b}\right], \\ 1+\frac{\log x}{\log b}+(1-x) \frac{1}{b-1}, & \text { если } x \in\left[\frac{1}{b}, 1\right] .\end{cases}
$$

ОПРЕДЕЛЕНИЕ 4. Для любого члена $x_{n}$ последовательности, $x_{n}=0.0 \ldots 0 a_{1} a_{2} \ldots$, где $a_{1}$ является первой ненулевой цифрой $x_{n}$, определим

- $\widetilde{x}_{n}=0 . a_{1} a_{2} \ldots$, т.е. первые нулевые знаки опускаются;

- $x_{n}^{*}=0.0 a_{1} a_{2} \ldots$, т.е. мы добавляем на первую позицию нулевой знак. 
Заметим, что если одна из трех последовательностей $x_{n}, \widetilde{x}_{n}$ и $x_{n}^{*}$ удовлетворяет сильному B.L., то все три последовательности $x_{n}, \widetilde{x}_{n}$ и $x_{n}^{*}$ одновременно удовлетворяют сильному B.L. ${ }^{2}$

ПримеР 2. По определению сильного B.L. последовательность $x_{n} \in(0,1), n=$ $1,2, \ldots$, и $\widetilde{x}_{n}, n=1,2, \ldots$, одновременно удовлетворяют сильному B.L. Поскольку $\widetilde{x}_{n} \in[1 / b, 1)$, то каждая $\widetilde{g}(x) \in G\left(\widetilde{x}_{n}\right)$ является непрерывной в $x=0$ и следовательно $x_{n}$ удовлетворяет B.L. тогда и только тогда, когда для любой $\widetilde{g}(x) \in G\left(\widetilde{x}_{n}\right)$ справедливо уравнение (3.1). Так как $\widetilde{g}(x)=0$ для $x \in[0,1 / b]$, то уравнение (3.1) имеет вид $x=1-\widetilde{g}\left(1 / b^{x}\right)+0$ при $x \in[0,1]$ и отсюда

$$
\widetilde{g}(x)= \begin{cases}0, & \text { если } x \in\left[0, \frac{1}{b}\right], \\ 1+\frac{\log x}{\log b}, & \text { если } x \in\left[\frac{1}{b}, 1\right] .\end{cases}
$$

Пример 3. A.d.f. $g^{*}(x)$ последовательности $x_{n}^{*}$ может быть вычислена по формуле $g^{*}(x)=\widetilde{g}\left(f^{-1}(x)\right)$, где $f(x)=x / b$. Таким образом, имеем

$$
g^{*}(x)= \begin{cases}0, & \text { если } x \in\left[0, \frac{1}{b^{2}}\right], \\ 2+\frac{\log x}{\log b}, & \text { если } x \in\left[\frac{1}{b^{2}}, \frac{1}{b}\right], \\ 1, & \text { если } x \in\left[\frac{1}{b}, 1\right]\end{cases}
$$

и для $g^{*}(x)$ справедливо уравнение (3.1). Аналогично, полагая $x_{n}^{* *}=0.00 a_{1} a_{2} \ldots$, a.d.f. последовательности $x_{n}^{* *}, n=1,2, \ldots$, есть

$$
g^{* *}(x)= \begin{cases}0, & \text { если } x \in\left[0, \frac{1}{b^{3}}\right], \\ 3+\frac{\log x}{\log b}, & \text { если } x \in\left[\frac{1}{b^{3}}, \frac{1}{b^{2}}\right], \\ 1, & \text { если } x \in\left[\frac{1}{b^{2}}, 1\right]\end{cases}
$$

и $g^{* *}(x)$ снова удовлетворяет уравнению (3.1).

A.d.f $\widetilde{g}(x)$ последовательности $\widetilde{x}_{n}, n=1,2, \ldots$, может быть вычислена непосредственно из a.d.f. $g(x)$ последовательности $x_{n}, n=1,2, \ldots$.

Теорема 6. Предположим, что $x_{n} \in(0,1)$ имеет непрерывную в $x=0$ a.d.f. $g(x)$. Тогда последовательность $\widetilde{x}_{n}$ имеет а.d.f. $\widetilde{g}(x)$ вида

$$
\widetilde{g}(x)=\sum_{i=0}^{\infty}\left(g\left(\frac{x}{b^{i}}\right)-g\left(\frac{1}{b^{i+1}}\right)\right)
$$

для $x \in[1 / b, 1]$ u $\widetilde{g}(x)=0$ для $x \in[0,1 / b]$.

\footnotetext{
${ }^{2}$ Для последовательности $x_{n} \in(0,1)$ набор всех d.f. $G\left(x_{n}\right)$ не зависит от основания $b$, по которому представлено $x_{n}$, а последовательность $\widetilde{x}_{n}$ зависит.
} 
ДоказАтЕЛьство. Любое $t \in\left[1 / b^{i+1}, 1 / b^{i}\right)$ имеет $i$ первых знаков равные нулю и отсюда $t b^{i} \in[1 / b, 1)$. Поэтому, для любого $x \in[1 / b, 1)$ имеем единственное $t=x / b^{i}$ такое, что $t \in\left[1 / b^{i+1}, 1 / b^{i}\right)$. Кроме того,

$$
\frac{\#\left\{n \leqslant N ; x_{n} \in\left[1 / b^{i+1}, x / b^{i}\right)\right\}}{N} \rightarrow g\left(\frac{x}{b^{i}}\right)-g\left(\frac{1}{b^{i+1}}\right),
$$

и выражение (3.13) следует из непрерывности $g(x)$ в $x=0$.

ПримеР 4. Пусть $x_{n}, n=1,2, \ldots$, есть последовательность на $(0,1)$ c a.d.f. $g(x)$, определенной из (3.9). По теореме 4 последовательность $x_{n}$ удовлетворяет сильному B.L. по основанию $b$. По теореме 6 , если $x \in[1 / b, 1]$, то мы имеем

$$
\begin{aligned}
\widetilde{g}(x) & =\sum_{i=0}^{\infty}\left(g\left(\frac{x}{b^{i}}\right)-g\left(\frac{1}{b^{i+1}}\right)\right)=g(x)-\frac{1}{b}+\left(x-\frac{1}{b}\right) \frac{1}{b}+\left(x-\frac{1}{b}\right) \frac{1}{b^{2}}+\cdots \\
& =1+\log _{b} x+(1-x) \frac{1}{b-1}-\frac{1}{b}+\left(x-\frac{1}{b}\right) \frac{1}{b-1},
\end{aligned}
$$

что обеспечивает равенство $1+\log _{b} x$ и $(3.10)$, что и ожидается.

Из примера 2 непосредственно следует

Теорема 7. Последовательность $x_{n} \in(0,1), n=1,2, \ldots$, удовлетворяет сильному B.L. по основанию $b$ тогда и только тогда, когда $\widetilde{x}_{n}, n=1,2, \ldots$, uмеет a.d.f. вида (3.10). Кроме того, если последовательность $x_{n} \in(0,1)$ имеет a.d.f. (3.10), то эту a.d.f. имеет также $\widetilde{x}_{n}$.

Следующая теорема упрощает функциональное уравнение (3.1) в теореме 4.

Теорема 8. Предположим, что d.f. $g(x) \in G\left(x_{n}\right)$ непрерывна в $x=0$. Тогда $x_{n}$ удовлетворяет силъному B.L. по основанию $b$ толъко тогда, когда

$$
1+\log _{b} x=\sum_{i=0}^{\infty}\left(g\left(\frac{x}{b^{i}}\right)-g\left(\frac{1}{b^{i+1}}\right)\right) \quad \text { npu } \quad x \in\left[\frac{1}{b}, 1\right]
$$

для любой d.f. $g(x) \in G\left(x_{n}\right)$.

ДокАЗАТЕЛЬСтво. Предположим d.f. $g(x) \in G\left(x_{n}\right)$ непрерывна в $x=0$ и $F_{N_{i}}(x) \rightarrow$ $g(x)$ при $i \rightarrow \infty$ (см. (2.5)). Аналогично описанному в теореме 6 последовательность $\widetilde{x}_{n}$ имеет d.f. $\widetilde{g}(x)$ вида (3.13) для той же последовательности индексов $N_{i}$. Из теоремы 7 следует, что $\widetilde{g}(x)$ для любой последовательности индексов $N_{i}$ должна иметь вид (3.10), который обеспечивает (3.15).

Альтернативное доказательство следует из (3.1), превращая $b^{x}$ в $x$.

Как можно видеть из следующих примеров 5 и 6 , существует последовательность целых чисел, удовлетворяющая сильному B.L. для произвольного основания $b$, но это не справедливо для последовательностей $x_{n} \in(0,1)$.

ПримеР 5. Согласно [10; с. 2-117, 2.12.14] последовательность

$$
\alpha n \log ^{\tau} n \bmod 1, \quad \alpha \neq 0, \quad 0<\tau \leqslant 1,
$$

является u.d. Отсюда следует, что $x_{n}=n^{n}$ удовлетворяет сильному B.L. для произвольного целого основания $b$, поскольку

$$
\log _{b} n^{n}=n \log n \frac{1}{\log b} .
$$


В этом случае последоваельность обратных значений $1 / x_{n} \in(0,1)$ имеет a.d.f. $c_{0}(x)$, которая разрывна в $x=0$.

ПримеР 6. Согласно [10; с. 2-117, 2.12.15] последовательность

$$
\alpha n^{2} \log ^{\tau} n \bmod 1, \quad \alpha \neq 0, \quad 0<\tau \leqslant 1,
$$

является u.d. Отсюда следует, что $x_{n}=n^{n^{2}}$ удовлетворяет сильному B.L. для произвольного целого основания $b$. И снова последовательность обратных значений $1 / x_{n}$ имеет a.d.f. $c_{0}(x)$.

ПримеР 7. Последовательность $x_{n}=b^{\{n \alpha\}}, n=1,2, \ldots$, где $\alpha$ является иррациональным, удовлетворяет сильному B.L. по основанию $b$, но не сильному по основанию $b^{r}$ при $r \geqslant 2$, так как

$$
\log _{b^{r}} b^{\{n \alpha\}}=\frac{\{n \alpha\}}{r} \in\left[0, \frac{1}{r}\right) .
$$

Более того, $x_{n}$ не удовлетворяет B.L. по основанию $b^{r}$ порядка 1 , потому что каждая d.f. $g(x)$ последовательности $\{n \alpha\} / r$ является константой на $[1 / r, 1]$, и при $x=$ $0 .(b-1)(b-1) \ldots(b-1)$ имеем $1-x=1 / b^{r}$. Таким образом, $g(x) \neq x$, и критерий теоремы 1 не выполняется.

В следующих примерах мы можем видеть, что существует последовательность $x_{n} \in(0,1)$, которая удовлетворяет B.L. по конечному набору различных оснований.

ПримеР 8. Пусть $x_{n} \in(0,1)$ есть последовательность с a.d.f. $g_{1}(x)$ вида $(3.10)$, т.е.

$$
g_{1}(x)= \begin{cases}0, & \text { если } x \in\left[0, \frac{1}{b_{1}}\right], \\ 1+\log _{b_{1}} x, & \text { если } x \in\left[\frac{1}{b_{1}}, 1\right] .\end{cases}
$$

Тогда согласно примеру 2 эта последовательность удовлетворяет сильному B.L. по $b_{1}$. Вычислим теперь все основания $b$, для которых $x_{n}$ в то же время удовлетворяет B.L. Каждое такое $b$ должно удовлетворять $(3.15)$ с $g(x)=g_{1}(x)$ и при $x \in[1 / b, 1]$. Что обеспечивает неравенство (3.22) (см. ниже в доказательстве теоремы 9) вида

$$
1+\log _{b} x \leqslant g_{1}(x) \leqslant 1+\log _{b} x+g_{1}\left(\frac{1}{b}\right) .
$$

a) Пусть $b_{1}<b$. Тогда при $x \in\left[1 / b, 1 / b_{1}\right]$ имеем $g_{1}(x)=0$, что противоречит (3.22).

b) Пусть $b<b_{1}$. Предположим, что $k$ есть первое положительное целое такое, что

$$
\frac{1}{b^{k}} \leqslant \frac{1}{b_{1}}<\frac{1}{b^{k-1}} \text {. }
$$

Тогда (3.15) имеет вид

$$
1+\log _{b} x=\sum_{i=0}^{k-1}\left(g_{1}\left(\frac{x}{b^{i}}\right)-g_{1}\left(\frac{1}{b^{i+1}}\right)\right),
$$

где

$$
g_{1}\left(\frac{x}{b^{i}}\right)-g_{1}\left(\frac{1}{b^{i+1}}\right)=\left(1+\log _{b_{1}} \frac{x}{b^{i}}\right)-\left(1+\log _{b_{1}} \frac{1}{b^{i+1}}\right)
$$


$i=1,2, \ldots, k-2$. При $i=k-1$ имеем $g_{1}\left(1 / b^{k}\right)=0$, и

$$
g_{1}\left(\frac{x}{b^{k-1}}\right)=1+\log _{b_{1}} \frac{x}{b^{k-1}} \quad \text { для } \quad x \in\left[\frac{b^{k-1}}{b_{1}}, 1\right] .
$$

Таким образом, (3.19) имеет вид

$$
1+\log _{b} x=\log _{b_{1}}\left(x \cdot \frac{x}{b} \ldots \frac{x}{b^{k-1}}\right)-\log _{b_{1}}\left(\frac{1}{b} \cdots \frac{1}{b^{k-1}}\right)+1=1+\log _{b_{1}} x^{k},
$$

и уравнение (3.20) справедливо тогда и только тогда, когда

$$
\frac{\log x}{\log b}=\frac{k \log x}{\log b_{1}},
$$

что эквивалентно $b_{1}=b^{k}$. В случае $b_{1}=b^{k}$ имеем

$$
x \in\left[\frac{b^{k-1}}{b_{1}}, 1\right]=\left[\frac{1}{b}, 1\right]
$$

и, следовательно, $x_{n}, n=1,2, \ldots$, удовлетворяет сильному B.L. также для оснований $b$, которые являются целыми корнями $b_{1}$.

Получим аналогичный результат также для d.f. вида (3.9).

ПримеР 9. Пусть $x_{n}, n=1,2, \ldots$, есть последовательность на $(0,1)$ c a.d.f. $g(x)$, определяемой из (3.9) при $b=b_{1}$, т.е.

$$
g_{1}(x)= \begin{cases}x, & \text { если } x \in\left[0, \frac{1}{b_{1}}\right], \\ 1+\log _{b_{1}}+(1-x) \frac{1}{b_{1}-1}, & \text { если } x \in\left[\frac{1}{b_{1}}, 1\right] .\end{cases}
$$

Тогда по теореме 4 последовательность $x_{n}$ удовлетворяет сильному B.L. по основанию $b_{1}$. Далее мы найдем все другие основания $b$, для которых $x_{n}$ снова удовлетворяет сильному B.L. Каждое такое $b$ должно удовлетворять $(3.15)$ с $g(x)=g_{1}(x)$ при $x \in[1 / b, 1]$.

a) Пусть $b_{1}<b$. Тогда при $x \in[1 / b, 1]$ имеем

$$
\frac{x}{b^{i}}, \frac{1}{b^{i+1}} \in\left[0, \frac{1}{b_{1}}\right] \quad \text { для } i=1,2, \ldots,
$$

и уравнение (3.15) имеет вид

$$
1+\log _{b} x=g_{1}(x)-g_{1}\left(\frac{1}{b}\right)+\sum_{i=1}^{\infty}\left(\frac{x}{b^{i}}-\frac{1}{b^{i+1}}\right)
$$

а при $x \in\left[1 / b, 1 / b_{1}\right]$ имеем $g_{1}(x)-g_{1}(1 / b)=x-1 / b$ и

$$
1+\log _{b} x=\left(x-\frac{1}{b}\right) \frac{b}{b-1} .
$$

Противоречие. 
b) Пусть $b<b_{1}$, и пусть $k$ - такое целое, что

$$
\frac{1}{b^{k}} \leqslant \frac{1}{b_{1}}<\frac{1}{b^{k-1}}
$$

Во всех случаях $x \in[1 / b, 1]$ влечет

$$
\frac{x}{b^{i}} \in\left[\frac{1}{b^{i+1}}, \frac{1}{b^{i}}\right] .
$$

Для случая b) имеем

(i) на интервалах $\left[1 / b^{i+1}, 1 / b^{i}\right], i=1,2, \ldots, k-2$, d.f. $g_{1}(x)$ имеет вид

$$
g_{1}(x)=1+\log _{b_{1}} x+(1-x) \frac{1}{b_{1}-1}
$$

(ii) тот же вид $g_{1}(x)$ имеем также на $\left[1 / b_{1}, 1 / b^{k-1}\right]$;

(iii) на интервалах $\left[1 / b^{i+1}, 1 / b^{i}\right], i=k, k+1, \ldots$, d.f. $g_{1}(x)=x$.

Пользуясь (i)-(iii) для

$$
g_{1}\left(\frac{x}{b^{i}}\right), \quad g_{1}\left(\frac{1}{b^{i+1}}\right), \quad g_{1}\left(\frac{x}{b^{k-1}}\right), \quad g_{1}\left(\frac{1}{b^{k}}\right),
$$

находим (3.15) вида

$$
\begin{array}{rl}
1+\log _{b}(x)=\log _{b_{1}} & x \frac{x}{b} \cdots \frac{x}{b^{k-2}}-\log _{b_{1}} \frac{1}{b} \cdots \frac{1}{b^{k-1}} \\
& -\frac{x}{b_{1}-1}\left(1+\frac{1}{b}+\cdots+\frac{1}{b^{k-2}}\right)+\frac{1}{b_{1}-1}\left(\frac{1}{b}+\cdots+\frac{1}{b^{k-1}}\right) \\
& +\left(1+\log _{b_{1}} \frac{x}{b^{k-1}}+\left(1-\frac{x}{b^{k-1}}\right) \frac{1}{b_{1}-1}\right)-\frac{1}{b^{k}} \\
& +\frac{x}{b^{k}} \frac{1}{1-1 / b}-\frac{1}{b^{k+1}} \frac{1}{1-1 / b} .
\end{array}
$$

Просуммировав получим, что для $x / b^{k-1} \in\left[1 / b_{1}, 1 / b^{k-1}\right]$ соотношение

$$
1+\log _{b} x=1+\log _{b_{1}} x^{k}+(1-x) \frac{b\left(b^{k}-1\right)}{\left(b_{1}-1\right)(b-1) b^{k}}-(1-x) \frac{b}{(b-1) b^{k}},
$$

справедливо только, если $b_{1}=b^{k}$. В этом случае

$$
\frac{x}{b^{k-1}} \in\left[\frac{1}{b_{1}}, \frac{1}{b^{k-1}}\right] \quad \Longleftrightarrow \quad x \in\left[\frac{b^{k-1}}{b_{1}}, 1\right] \quad \Longleftrightarrow \quad x \in\left[\frac{1}{b}, 1\right]
$$

и (3.15) справедливо. Таким образом, последовательность $x_{n}$ удовлетворяет сильному B.L. также для основания $b=\sqrt[k]{b_{1}}$.

Примеры 8 и 9 дали импульс к следующей теореме.

Теорема 9. Предположим, что для последовательности $x_{n} \in(0,1), n=1,2, \ldots$, каждая d.f. $g(x) \in G\left(x_{n}\right)$ является непрерьвной в $x=0$. Тогда существует лишь конечно много различных цельх оснований $b$, для которых последовательность $x_{n}$ одновременно удовлетворяет сильному В.L. Более того, если последовательность $x_{n}$ удовлетворяет сильному B.L. по основанию $b, u$ для некоторого $k=1,2, \ldots$ существует $k$-й иельй корень $\sqrt[k]{b}$, то $x_{n}$ удовлетворяет сильному B.L. также по основанию $\sqrt[k]{b}$. 
ДокАЗАТЕЛЬСтво. $1^{\circ}$. Предположим, что $x_{n}$ удовлетворяет сильному B.L. по основанию $b$, и пусть $g(x) \in G\left(x_{n}\right)$. Тогда по теореме 8 при $x \in[1 / b, 1]$ имеем $(3.15)$. Перепишем его в виде

$$
g(x)=1+\log _{b} x+g\left(\frac{1}{b}\right)-\sum_{i=1}^{\infty}\left(g\left(\frac{x}{b^{i}}\right)-g\left(\frac{1}{b^{i+1}}\right)\right)
$$

отсюда

$$
1+\log _{b} x \leqslant g(x) \leqslant 1+\log _{b} x+g\left(\frac{1}{b}\right) .
$$

Если последовательность $x_{n}$ удовлетворяет сильному B.L. для бесконечно многих $b$, то (3.22) влечет $g(x)=1$ при $x \in(0,1]$ - противоречие. Количественно, если $g\left(x_{0}\right)<1$, то или $x_{0}<1 / b$, т.е. $b<1 / x_{0}$, или $1 / b \leqslant x_{0}$, и из $(3.22)$ имеем $1+\log _{b} x_{0} \leqslant$ $g\left(x_{0}\right)$, а тогда

$$
1-g\left(x_{0}\right) \leqslant \frac{-\log x_{0}}{\log b}
$$

откуда

$$
b \leqslant \max \left(\frac{1}{x_{0}}, e^{-\log x_{0} /\left(1-g\left(x_{0}\right)\right)}\right) .
$$

$2^{\circ}$. Теперь предположим, что $x_{n}$ удовлетворяет сильному B.L. по основанию $b$, т.е. последовательность $\log _{b} x_{n} \bmod 1$ является u.d. Применяя известный критерий Вейля, имеем следующую цепь последствий:

(i) имеем

$$
\frac{1}{N} \sum_{n=1}^{N} e^{2 \pi i h \log _{b} x_{n}} \rightarrow 0 \quad \text { при } \quad N \rightarrow \infty
$$

и при каждом $h=1,2, \ldots$, поэтому

(ii) имеем

$$
\frac{1}{N} \sum_{n=1}^{N} e^{2 \pi i h k \log _{b} x_{n}} \rightarrow 0 \quad \text { при } \quad N \rightarrow \infty
$$

и при каждом $h, k=1,2, \ldots$ и, следовательно, получим, что

(iii) $x_{n}^{k}$ снова удовлетворяет сильному B.L. по основанию $b$;

(iv) таким образом, если $g(x) \in G\left(x_{n}\right)$, то $g(\sqrt[k]{x}) \in G\left(x_{n}^{k}\right)$ и наоборот, поскольку

$$
x_{n}^{k}<x \quad \Longleftrightarrow \quad x_{n}<\sqrt[k]{x} ;
$$

(v) далее, для каждой непрерывной в $x=0$ d.f. $g(x), g(x)$ и $g(\sqrt[k]{x}), k=1,2, \ldots$, одновременно разрешают (3.15).

Следовательно, (3.15) влечет

$$
1+\log _{b} x=\sum_{i=0}^{\infty}\left(g\left(\sqrt[k]{\frac{x}{b^{i}}}\right)-g\left(\sqrt[k]{\frac{1}{b^{i+1}}}\right)\right) \quad \text { для } \quad x \in\left[\frac{1}{b}, 1\right] .
$$

Пользуясь для упрощения соотношением

$$
\sum_{i=0}^{\infty}\left(g\left(\frac{1}{b^{i}}\right)-g\left(\frac{1}{b^{i+1}}\right)\right)=1,
$$


перепишем (3.15) в виде

$$
1+\log _{b} x=1-\sum_{i=0}^{\infty}\left(g\left(\frac{1}{b^{i}}\right)-g\left(\frac{x}{b^{i}}\right)\right) \quad \text { при } \quad x \in\left[\frac{1}{b}, 1\right],
$$

а (3.24) - в виде

$$
1+\log _{b} x=1-\sum_{i=0}^{\infty}\left(g\left(\sqrt[k]{\frac{1}{b^{i}}}\right)-g\left(\sqrt[k]{\frac{x}{b^{i}}}\right)\right) \quad \text { для } \quad x \in\left[\frac{1}{b}, 1\right] .
$$

Пусть для целого $k$ у нас есть целый корень $\sqrt[k]{b}$. Пользуясь соотношениями

$$
\begin{aligned}
& \text { (i) } 1+\log _{b} x=1+\log _{\sqrt[k]{b}} \sqrt[k]{x} \\
& \text { (ii) } g\left(\sqrt[k]{\frac{1}{b^{i}}}\right)-g\left(\sqrt[k]{\frac{x}{b^{i}}}\right)=g\left(\frac{1}{(\sqrt[k]{b})^{i}}\right)-g\left(\frac{\sqrt[k]{x}}{(\sqrt[k]{b})^{i}}\right) ; \\
& \text { (iii) } x \in\left[\frac{1}{b}, 1\right] \quad \Longleftrightarrow \quad \sqrt[k]{x} \in\left[\frac{1}{\sqrt[k]{b}}, 1\right]
\end{aligned}
$$

и делая замену $\sqrt[k]{x} \rightarrow x$, получим (3.26) в виде

$$
1+\log _{\sqrt[k]{b}} x=1-\sum_{i=0}^{\infty}\left(g\left(\frac{1}{(\sqrt[k]{b})^{i}}\right)-g\left(\frac{x}{(\sqrt[k]{b})^{i}}\right)\right) .
$$

Согласно теореме 8 последовательность $x_{n}$ удовлетворяет сильной B.L. по основанию $\sqrt[k]{b}$.

4. Простые следствия теории равномерного распределения. Дьяконис [4] и Павлов [11] были первыми, кто применил теорию равномерного распределения к закону Бенфорда (B.L.). Например, Дьяконис, пользуясь критерием Кеннеди (см. [10; с. 2-13, 2.2.9]), доказал следующую теорему.

Теорема 10. Если последовательность $x_{n}>0, n=1,2, \ldots$, удовлетворяет силъному B.L. по основанию b, то

$$
\limsup _{n \rightarrow \infty} n\left|\log \frac{x_{n+1}}{x_{n}}\right|=\infty
$$

Павлов доказал посредством разностной теоремы Ван дер Корпута [8; с. 26, теорема 3.1] следующее утверждение.

Теорема 11. Пусть $x_{n}>0, n=1,2, \ldots$. Если для каждого $k=1,2, \ldots$ последовательность отношений $x_{n+k} / x_{n}, n=1,2, \ldots$, удовлетворяет сильному B.L. по основанию $b$, то первоначальная последовательность $x_{n}, n=1,2, \ldots$, также удовлетворяет силъному B.L. по основанию $b$.

Для полноты далее представим несколько других приложений.

Теорема 12. Положительные последовательности $x_{n} u 1 / x_{n}, n=1,2, \ldots$, удовлетворяют сильному B.L. по основанию $b$ одновременно.

ДокАЗАТЕЛЬСтво. Обе последовательности $u_{n}$ и $-u_{n}$ являются u.d. $\bmod 1$ одновременно, поскольку их суммы Вейля являются комплексно сопряженными в отношении друг друга. 
Теорема 13. Положительные последовательности $x_{n}$ u $n x_{n}, n=1,2, \ldots$, удовлетворяют сильному B.L. по основанию $b$ одновременно.

ДокАЗАТЕЛЬСТво. Обе последовательности $u_{n}$ и $u_{n}+\log n$ являются u.d. $\bmod 1$ одновременно (см. [10; с. 2-27, 2.3.6.]).

Теорема 14. Предположим, что последовательность $0<x_{1} \leqslant x_{2} \leqslant \cdots$ удовлетворяют сильному B.L. по иелому основанию $b>1$. Тогда

$$
\lim _{n \rightarrow \infty} \frac{\log x_{n}}{\log n}=\infty
$$

ДокАЗАТЕЛЬСтво. Из теоремы Нидеррайтера следует [10; с. 2-12, 2.2.8]: каждая монотонная u.d. последовательность $u_{n} \bmod 1$ должна удовлетворять

$$
\lim _{n \rightarrow \infty} \frac{\left|u_{n}\right|}{\log n}=\infty
$$

Здесь достаточно положить $u_{n}=\log x_{n}$ вместо $u_{n}=\log _{b} x_{n}$.

Теорема 15. Для последовательности $x_{n}>0, n=1,2, \ldots$, предположим, что

(i) $\lim _{n \rightarrow \infty} x_{n}=\infty$ монотонно;

(ii) $\lim _{n \rightarrow \infty} \log \left(x_{n+1} / x_{n}\right)=0$ монотонно.

Тогда последователъность $x_{n}$ удовлетворяет сильному B.L. по любому основанию $b$ только тогда, когда

$$
\lim _{n \rightarrow \infty} n \log \frac{x_{n+1}}{x_{n}}=\infty .
$$

ДокАЗАТЕЛЬСтво. Доказательство следует из разностной теоремы Фейерса в виде из [10; с. 2-13, 2.2.11].

Утверждение [10; с. 2-14, 2.2.12] влечет следующее.

ТЕОРема 16. Пусть $x_{n}>0$ - последовательность, которая удовлетворяет соотношению

$$
\lim _{n \rightarrow \infty} \log _{b} \frac{x_{n+1}}{x_{n}}=\theta,
$$

где $\theta$ является иррациональным. Тогда $x_{n}$ удовлетворяет сильному B.L. по основанию $b$.

Можно также изучать асимптотическую плотность первоначальных цифр последовательностей без B.L.

Пример 10. Известно, что возрастающая последовательность всех натуральных чисел $1,2,3, \ldots$ не удовлетворяет сильному B.L. (так же, как и простому B.L.) по любому основанию $b \geqslant 2$. Что следует из того факта, что $\log _{b} n \bmod 1$ не является u.d. Для плотности $n$, для которой $r$ первоначальных цифр - это $K=k_{1} k_{2} \ldots k_{r}$, Павлов [11] доказал, что

$$
\begin{aligned}
& \liminf _{N \rightarrow \infty} \frac{\#\{n \leqslant N ; n \text { имеет первых } r \text { цифр }=K\}}{N}=\frac{1}{K(b-1)}, \\
& \limsup _{N \rightarrow \infty} \frac{\#\{n \leqslant N ; n \text { имеет первых } r \text { цифр }=K\}}{N}=\frac{b}{(K+1)(b-1)} .
\end{aligned}
$$


Добавим следующее: по Пойя и Сегё [13] d.f. функция $\log _{b} n \bmod 1$ есть функция вида

$$
g_{u}(x)=\frac{b^{\min (x, u)}-1}{b^{u}}+\frac{1}{b^{u}} \frac{b^{x}-1}{b-1},
$$

где параметр $u$ пробегает $[0,1]$. Согласно Джулиано и Штрауху [14] для возрастающей последовательности $N_{i}, i=1,2, \ldots$, имеем (см. (2.4)) импликацию

$$
\log _{b} N_{i} \bmod 1 \rightarrow u \quad \Longrightarrow \quad F_{N_{i}}(x) \rightarrow g_{u}(x)
$$

и, следовательно,

$$
\frac{\#\left\{n \leqslant N_{i} ; n \text { имеет первых } r \text { знаков }=K\right\}}{N_{i}} \rightarrow g_{u}\left(x_{2}\right)-g_{u}\left(x_{1}\right)
$$

при $i \rightarrow \infty$, где

$$
x_{1}=\log _{b}\left(k_{1} \cdot k_{2} k_{3} \ldots k_{r}\right), \quad x_{2}=\log _{b}\left(k_{1} \cdot k_{2} k_{3} \ldots\left(k_{r}+1\right)\right) .
$$

Теперь результаты Павлова (4.4) и (4.5) следуют из

$$
\begin{gathered}
\liminf _{N \rightarrow \infty} \frac{\#\{n \leqslant N ; n \text { имеет первых } r \text { цифр }=K\}}{N}=\min _{u \in[0,1]}\left(g_{u}\left(x_{2}\right)-g_{u}\left(x_{1}\right)\right), \\
\limsup _{N \rightarrow \infty} \frac{\#\{n \leqslant N ; n \text { имеет первых } r \text { цифр }=K\}}{N}=\max _{u \in[0,1]}\left(g_{u}\left(x_{2}\right)-g_{u}\left(x_{1}\right)\right),
\end{gathered}
$$

где в $u=x_{1}$ появляется минимум, а в $u=x_{2}$ - максимум. Далее, если $N_{i}=\left[b^{i+u}\right]$, то $\log _{b} N_{i} \bmod 1 \rightarrow u$ при $i \rightarrow \infty$.

Заметим, что Павлов сформулировал свой результат для более общей последовательности $a n^{\alpha}, n=1,2, \ldots$, где $a$ и $\alpha$ - фиксированные положительные вещественные числа. Полагая

$$
f(x)=c_{1}+c_{2} \log x, \quad \text { где } \quad c_{1}=\frac{\log a}{\log b}, \quad c_{2}=\frac{\alpha}{\log b},
$$

имеем $\log _{b} x_{n}=f(n)$ и, применяя теорему 1 из [14], отсюда находим, что любая d.f. $g_{u}(x) \in G\left(\log _{b} x_{n}\right)$ имеет вид

$$
g_{u}(x)=\frac{b^{\min (x, u) / \alpha}-1}{b^{u / \alpha}}+\frac{1}{b^{u / \alpha}} \frac{b^{x / \alpha}-1}{b^{1 / \alpha}-1},
$$

где параметр $u$ пробегает $[0,1]$ и для возрастающей последовательности $N_{i}, i=$ $1,2, \ldots$, имеем

$$
\frac{\log a}{\log b}+\alpha \frac{\log N_{i}}{\log b} \bmod 1 \rightarrow u \quad \Longrightarrow \quad F_{N_{i}}(x) \rightarrow g_{u}(x) .
$$

Теперь, вычисляя производную $d\left(g_{u}\left(x_{2}\right)-g_{u}\left(x_{1}\right)\right) / d u$ в $(4.8)$, мы можем видеть, что ее функция сигнум равна '-' при $u \in\left[0, x_{1}\right)$, постоянная ('+' или '-') на $\left(x_{1}, x_{2}\right)$ и '-' - на $\left(x_{2}, 1\right)$. Следовательно, $\min _{u \in[0,1]}\left(g_{u}\left(x_{2}\right)-g_{u}\left(x_{1}\right)\right)$ может появиться на $u=x_{1}, x_{2}, 1$ и $\max _{u \in[0,1]}\left(g_{u}\left(x_{2}\right)-g_{u}\left(x_{1}\right)\right)$ на $u=0, x_{2}$. Путем непосредственного вычисления находим результат Павлова

$$
\begin{aligned}
& \min _{u \in[0,1]}\left(g_{u}\left(x_{2}\right)-g_{u}\left(x_{1}\right)\right)=g_{x_{1}}\left(x_{2}\right)-g_{x_{1}}\left(x_{1}\right)=\frac{1}{b^{1 / \alpha}-1} \frac{(K+1)^{1 / \alpha}-K^{1 / \alpha}}{K^{1 / \alpha}} \\
& \max _{u \in[0,1]}\left(g_{u}\left(x_{2}\right)-g_{u}\left(x_{1}\right)\right)=g_{x_{2}}\left(x_{2}\right)-g_{x_{1}}\left(x_{2}\right)=\frac{b^{1 / \alpha}}{b^{1 / \alpha}-1} \frac{(K+1)^{1 / \alpha}-K^{1 / \alpha}}{(K+1)^{1 / \alpha}} .
\end{aligned}
$$




\section{СПИСОК ЦИТИРОВАННОЙ ЛИТЕРАТУРЫ}

[1] S. Newcomb, "Note on the frequency of use of the different digits in natural numbers", Amer. J. Math., 4 (1881), 39-40.

[2] F. Benford, "The law of anomalous numbers", Proc. Amer. Phil. Soc., 78 (1938), 551-572.

[3] R. A. Raimi, "The first digit problem", Amer. Math. Monthly, 83:7 (1976), 521-538.

[4] P. Diaconis, "The distribution of leading digits and uniform distribution mod 1", Ann. Probab., 5:1 (1977), 72-81.

[5] S. Kunoff, "N! has the first digit property", Fibonacci Quart., 25:4 (1987), 365-367.

[6] P. Schatte, "On mantissa distribution in computing and Benford's law", J. Inform. Process. Cybernet., 24:9 (1988), 443-455.

[7] K. Nagasaka, S. Kanemistu, J.-S. Shiue, "Benford's law: the logarithmic law of first digit", Number Theory. v. 1: Elementary and Analytic (Budapest, 1987), Colloq. Math. Soc. János Bolyai, 51, North-Holland, Amsterdam, 1990, 361-391.

[8] L. Kuipers, H. Niederreiter, Uniform Distribution of Sequences., Pure Appl. Math., John Wiley \& Sons, New York, 1974.

[9] M. Drmota, R.F. Tichy, Sequences, Discrepancies and Applications, Lecture Notes in Math., 1651, Springer-Verlag, Berlin, 1997.

[10] O. Strauch, Š. Porubský, Distribution of Sequences: A Sampler, Schr. Slowak. Akad. Wiss., 1, Peter Lang, Frankfurt am Main, 2005.

[11] А.И. Павлов, "О распределении дробных долей и законе Ф. Бенфорда", Изв. АН CССР. Сер. матем., 45:4 (1981), 760-774.

[12] P. Kostyrko, M. Mačaj, T. Šalát, O. Strauch, "On statistical limit points", Proc. Amer. Math. Soc., 129:9 (2001), 2647-2654.

[13] G. Pólya, G. Szegő, Aufgaben und Lehrsätze aus der Analysis. Band 1, 2, Grundlehren Math. Wiss., 19, Springer-Verlag, Berlin, 1964.

[14] A.R. Giuliano, O. Strauch, "On weighted distribution functions of sequences", Unif. Distrib. Theory, 3:1 (2008), 1-18.

\section{В. Балаж}

Словацкий технический университет, Братислава, Словакия

E-mail: vladimir.balaz@stuba.sk

\section{K. Нагасака}

Университет Хосей, Токио, Япония

E-mail: nagasaka@hosei.ac.jp

\section{О. Штраух}

Математический институт САН, Братислава, Словакия

E-mail: strauch@mat.savba.sk 